\title{
La gestion de la matière organique du sol, un enjeu important pour limiter la lixiviation du nitrate vers les eaux souterraines
}

\author{
Christophe Vandenberghe, Gilles Colinet
}

Université de Liège - Gembloux Agro-Bio Tech. Passage des Déportés, 2. BE-5030 Gembloux (Belgique). E-mail :

c.vandenberghe@ulg.ac.be

Reçu le 16 décembre 2015, accepté le 23 aout 2016.

Cet article est distribué suivant les termes et les conditions de la licence CC-BY (http://creativecommons.org/licenses/by/4.0/ deed.fr)

Description du sujet. La concentration en nitrate dans la moitié des masses d'eau souterraine de la région wallonne (Belgique) a justifié le classement de celles-ci en zone vulnérable, en application de la Directive Nitrates (DN). Cette zone vulnérable présente une diversité de contextes agro-pédologiques. Ainsi, le contenu en azote des sols agricoles peut localement varier d'un facteur 4.

Objectifs. L'étude consiste à établir si la teneur en carbone organique total (COT) d'un sol constitue un facteur de risque significatif quant à la lixiviation du nitrate vers les eaux souterraines.

Méthode. L'article s'appuie sur des mesures COT et de reliquat d'azote nitrique dans le sol en début de période de lixiviation (APL - Azote Potentiellement Lessivable), réalisées en 2012 dans 1500 parcelles lors d'un contrôle organisé en application du Programme de Gestion Durable de l'Azote, transposition de la DN en région wallonne.

Résultats. L'examen des résultats illustre d'une part que l'APL est positivement corrélé au COT et que les parcelles qualifiées de «non conformes » présentent une concentration moyenne en COT significativement supérieure aux autres résultats.

Conclusions. L'APL étant un indicateur environnemental du risque de lixiviation du nitrate, il apparait que le contenu COT est généralement de nature à constituer un risque de contamination des eaux souterraines par le nitrate. Il convient dès lors d'une part, d'améliorer la prise en compte de ce paramètre dans les conseils de fertilisation azotée et, d'autre part, dans le cas de récolte estivale, de gérer au mieux le sol par une mise en place efficiente de cultures pièges à nitrate.

Mots-clés. Agriculture durable, azote, nitrate, carbone, lixiviation, minéralisation du carbone, eau souterraine, qualité de l'eau, agriculture, Belgique.

The management of soil organic matter, an important issue in reducing nitrate leaching to groundwater

Description of the subject. The nitrate concentration in half of the groundwater bodies of the Walloon Region (Belgium) has justified their classification as vulnerable zones under the Nitrates Directive (ND). This vulnerable area has a relatively high diversity of agro-soil environments. Thus, the nitrogen content of agricultural soils can vary locally by a factor 4 .

Objectives. This study aimed to establish whether the carbon content of a soil is a significant risk factor in nitrate leaching to groundwater.

Method. The article is based on measurements of nitrate nitrogen residue (APL) and soil total organic carbon (TOC) at the beginning of the leaching period. The measurements were made in 2012 in 1,500 plots, during inspections organized by the Wallonia Public Service. This was as part of the application of the Program for the Sustainable Management of Nitrogen in Agriculture, the scheme for implementing the ND within the Walloon region.

Results. Examination of the results showed both that the APL was positively correlated to the TOC and that plots with "noncompliant" APL results had a significantly higher average TOC than the other plots.

Conclusions. Since APL is an environmental indicator of the risk of nitrate leaching, it appears that the TOC may constitute a risk of groundwater contamination by nitrate. It is therefore advisable to improve the inclusion of this parameter in advice regarding nitrogen fertilization and secondly, in the case of summer harvest, to better manage the sowing of catch crops.

Keywords. Sustainable agriculture, nitrogen, nitrate, carbon, nitrate leaching, carbon mineralization, ground water, water quality, agriculture, Belgium. 


\section{INTRODUCTION}

En région wallonne (Belgique), l'évolution de la qualité (concentration en nitrate) des eaux souterraines entre 1980 et 2000 a justifié la délimitation de zones vulnérables et la mise en œuvre d'un programme d'actions (Programme de Gestion Durable de l'Azote PGDA $^{1}$ ) en application de la Directive Nitrates (91/676/ CEE) (Vandenberghe et al., 2010 ; Vandenberghe et al., 2013a). Le suivi de la concentration en nitrate dans les eaux souterraines est réalisé par les producteurs d'eau potable dans les aquifères qu'ils sollicitent et par l'Institut Scientifique de Service Public (ISSeP) dans les aquifères non exploités. Des échantillons d'eau sont ainsi prélevés régulièrement dans près de 900 ouvrages (galeries, puits, piézomètres, etc.) et analysés en vue d'y mesurer, entre autres, la concentration en nitrate.

Le PGDA, mis en place en 2002 et déjà révisé à plusieurs reprises, fixe des règles en matière de gestion de l'azote (organique et minéral) et de successions culturales afin de limiter la lixiviation et le ruissellement du nitrate. Des contrôles sont organisés par le Service public de Wallonie pour vérifier la bonne mise en œuvre de ces règles par les agriculteurs. Le dosage du reliquat d'azote nitrique dans le sol $(0-90 \mathrm{~cm})$ en fin d'automne (azote potentiellement lessivable - APL) fait partie des outils de contrôle. Destain et al. (2010), Deneufbourg et al. (2013a) et Deneufbourg et al. (2013b) ont montré (respectivement avec l'isotope 15 de l'azote, avec des lysimètres à l'échelle de la parcelle ou à l'échelle d'un petit bassin versant) que l'APL constitue un indicateur environnemental de la lixiviation du nitrate vers les eaux souterraines.

Ainsi, chaque année, des échantillons de sol sont prélevés dans plus de $5 \%$ des exploitations agricoles situées en zone vulnérable et les résultats d'analyse sont comparés à des valeurs de référence (Vandenberghe et al., 2013b).

En 2012, les épandages d'engrais de ferme (fumier, lisier, compost, etc.) représentaient en moyenne un apport de l'ordre de $98 \mathrm{~kg} \mathrm{~N} \cdot \mathrm{ha}^{-1} \cdot \mathrm{an}^{-1}$ sur les sols agricoles (cultures et prairies) (Brahy et al., 2014), ce qui correspond à environ une quinzaine de tonnes de matière organique fraiche par hectare enfouie dans le sol chaque année.

Depuis plusieurs années, la thématique du carbone dans les sols fait l'objet d'un intérêt croissant, que ce soit pour la protection des sols (identifiée par l'Europe comme une des huit menaces ${ }^{2}$ ) ou la lutte contre le changement climatique (par exemple, Objectif « 4 pour $1000 »$ en France ${ }^{3}$ ).

\footnotetext{
${ }^{1} \mathrm{http}: / /$ environnement.wallonie.be/legis/Codeenvironnement/co deeaucoordonneR.html

${ }^{2} \mathrm{http}$ //eur-lex.europa.eu/legal-content/FR/TXT/PDF/?uri=CELE $\mathrm{X}: 52006 \mathrm{DC} 0231 \&$ from=EN, (16.02.2016).
}

En Wallonie, les sols agricoles sont suivis régulièrement depuis plusieurs dizaines d'années pour des paramètres tels que le carbone organique total et l'azote total (Colinet et al., 2005 ; Genot et al., 2012).

Les matières organiques (endogène et exogène) présentent des effets agronomiques et environnementaux positifs :

- pour la stabilité du sol et la limitation de l'érosion (Chenu et al., 2000 ; Bronick et al., 2005) ;

- pour la fourniture d'éléments nutritifs aux plantes (Nevens et al., 2005 ; Grignani et al., 2007 ; Maltas et al., 2013) ;

- pour la valorisation de «déchets» organiques anthropiques ou issus d'élevages ;

- pour la dégradation de produits phytopharmaceutiques utilisés pour la protection des cultures et la lutte contre les ravageurs (Mukherjee, 2009 ; Fenoll et al., 2011 ; Fenoll et al., 2014) ;

- pour le stockage de carbone (Buysse et al., 2013; D'Hose et al., 2014 ; Vanden Nest et al., 2014) dans la lutte contre le réchauffement climatique.

Il convient cependant d'évaluer si cette course vers plus de carbone dans les sols ne présente pas d'effets négatifs pour l'environnement. On sait par exemple que les stocks importants de matière organique dans les sols, lorsqu'ils minéralisent, peuvent engendrer des flux importants (en concentration et en durée) de nitrate comme, par exemple, lors du retournement de vieilles prairies (Whitmore et al., 1992). La présente étude vise à apporter quelques éléments de réflexion à ce sujet, plus spécifiquement sur la thématique du nitrate et de l'eau. Concrètement, on recherchera l'existence d'une relation entre le carbone organique total contenu dans le sol (COT) et l'APL exprimé en valeur absolue ( $\mathrm{kg} \mathrm{N}^{-\mathrm{NO}_{3}^{-}}$. $\mathrm{ha}^{-1}$ ) ou en valeur relative (par rapport à une référence).

\section{MATÉRIEL ET MÉTHODES}

\subsection{Sites d'étude}

En 2012, en application du PGDA, le Service public de Wallonie a commandé près de 1500 contrôles APL dans des parcelles agricoles afin d'y évaluer la qualité de la gestion de l'azote pratiquée. Ces 1500 parcelles sont principalement situées en zone vulnérable ${ }^{4}$ (Figure 1). Les cultures présentes dans ces parcelles étaient pour la moitié des céréales et pour un quart du maïs; la

\footnotetext{
${ }^{3} \mathrm{http} / / /$ agriculture.gouv.fr/rejoignez-linitiative-4-pour-1000, (16.02.2016).

${ }^{4} \mathrm{Au}$ sens de la DN, il s'agit des territoires où les eaux (de surface et souterraines) contiennent ou risquent de contenir plus de $50 \mathrm{mg} \cdot \mathrm{l}^{-1}$ de nitrate ou qui ont subi ou qui risquent de subir dans un avenir proche une eutrophisation.
} 
betterave, la pomme de terre, le colza, les légumes et prairies représentant le dernier quart (Figure 2). Les échantillons prélevés lors de ces contrôles ont été analysés pour doser l'azote nitrique dans la couche $0-90 \mathrm{~cm}$ et le carbone organique total dans la couche $0-30 \mathrm{~cm}$

\subsection{Méthodes}

Afin d'évaluer correctement le contenu en azote nitrique du sol, au moins 15 carottes de sol sont prélevées par parcelle pour constituer un échantillon composite (Vandenberghe et al., 2014). Les prélèvements sont

Contrôle APL 2012

\section{Qualification}

- Bon

Satisfaisant

- Limite

- Non conforme

Zones vulnérables
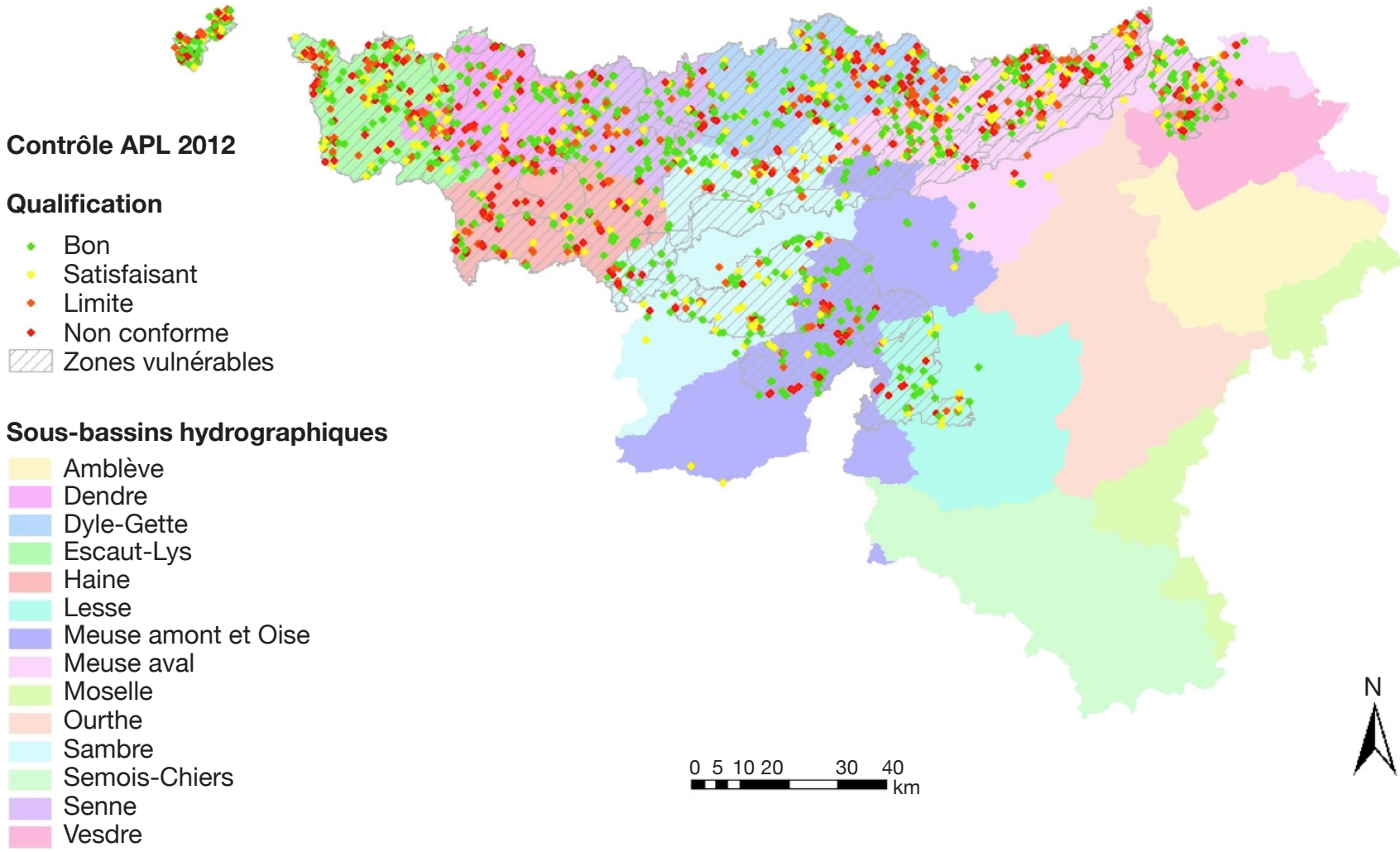

Figure 1. Spatialisation et qualification des résultats de contrôle de l'azote potentiellement lessivable (APL) en 2012 - Spatialization and qualification of the nitrate nitrogen residue (APL) results in 2012.
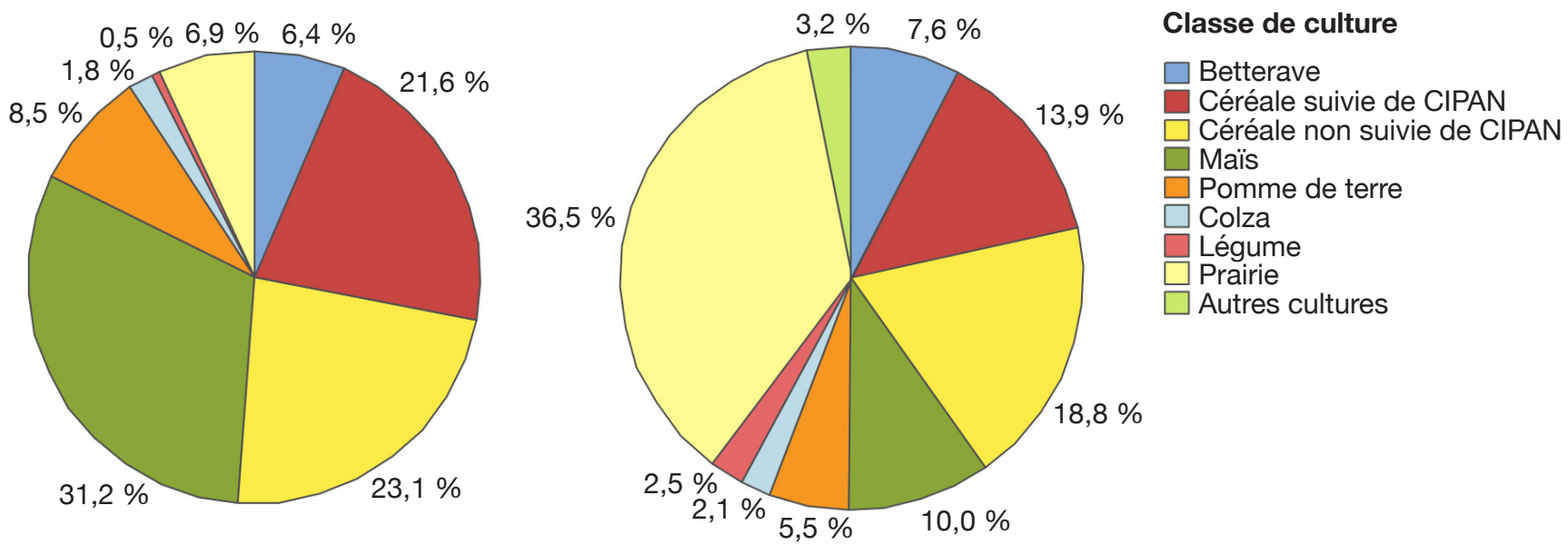

Figure 2. Distribution des cultures et prairies contrôlées lors de l'échantillonnage de l'azote potentiellement lessivable (APL) en 2012 (gauche) et des cultures et prairies présentes en zone vulnérable (droite) - Distribution of the crops and pasture controlled during the sampling of the nitrate nitrogen leaching (APL) in 2012 (left) and crops and pastures in the vulnerable zone (right). 
effectués dans trois couches successives de $30 \mathrm{~cm}$ pour atteindre ainsi la profondeur de $90 \mathrm{~cm}$. Ces échantillons sont conduits, dans un coffre thermiquement isolé, à un laboratoire agréé par le Service public de Wallonie. Ils sont analysés immédiatement ou stockés en chambre froide $\left(1{ }^{\circ} \mathrm{C}\right.$ à $\left.4{ }^{\circ} \mathrm{C}\right)$ pendant au maximum cinq jours avant analyse. Les échantillons, non séchés, sont homogénéisés par tamisage $(8 \mathrm{~mm})$ après quoi l'extraction de l'ion nitrate est effectuée sur une aliquote de minimum $30 \mathrm{~g}$ d'échantillon par une solution $\mathrm{KCl}$ de concentration $0,1 \mathrm{~N}$. Le rapport d'extraction (poids de terre/volume de solution d'extraction) est de $1 / 5^{\mathrm{e}}$. Les flacons sont placés dans un agitateur rotatif pendant $1 / 2 \mathrm{~h}$. La solution est ensuite laissée au repos pendant $1 / 2 \mathrm{~h}$ pour décantation. Le dosage du nitrate est effectué sur le surnageant qui, selon la méthode de dosage, est préalablement filtré ou centrifugé.

Si le dosage n'est pas effectué endéans les $3 \mathrm{~h}$ qui suivent l'extraction, les extraits sont stockés en chambre froide, à une température maximale de $4{ }^{\circ} \mathrm{C}$, à l'abri de la lumière pendant une durée maximale de $48 \mathrm{~h}$ ou sont congelés.

Le dosage est effectué selon la méthode colorimétrique de réduction du nitrate en nitrite (à l'aide notamment de cadmium) avec dosage de l'ion nitrite par la réaction de Griess-Ilosvay modifiée (Bremner, 1965 ; Guiot, 1975).

Le résultat obtenu est exprimé en $\mathrm{kg} \mathrm{N}-\mathrm{NO}_{3}^{-} \cdot \mathrm{ha}^{-1}$ (sur $90 \mathrm{~cm}$ de profondeur) en tenant compte :

- de la teneur en eau de l'échantillon de sol $(24 \mathrm{~h}$ de séchage à $105^{\circ} \mathrm{C}$ ) ;

- d'une densité de $1,35 \mathrm{~g} \cdot \mathrm{cm}^{-3}$ pour la couche $0-30 \mathrm{~cm}$, de $1,5 \mathrm{~g} \cdot \mathrm{cm}^{-3}$ pour les deux couches sous-jacentes ;

- de la charge caillouteuse renseignée par la Carte Numérique des Sols de Wallonie (Bah et al., 2007).

Chaque résultat est ensuite comparé à une référence établie sur base d'observations réalisées en situation de gestion optimale de l'azote (Vandenberghe et al., 2004 ; Vandenberghe et al., 2005; Vandenberghe et al., 2013b) pour pouvoir être qualifié selon quatre catégories (bon, satisfaisant, limite ou non conforme).

Le dosage du carbone dans les échantillons prélevés dans la couche 0-30 cm a été effectué par combustion sèche (norme ISO 10694 de 1995) ou par la méthode de Walkley Black (dérivée de la norme ISO 14235 de 1998). Le résultat obtenu est exprimé en \% carbone organique total (\%COT), soit g COT $100 \mathrm{~g}^{-1}$ sol.

Enfin, les propriétés édaphiques (texture, hydromorphie) de chaque parcelle échantillonnée ont été extraites de la Carte Numérique des Sols de Wallonie.

L'étude aborde les résultats de trois manières différentes. Dans un premier temps (\$3.1.), on observe culture par culture l'existence d'une relation entre le $\%$ COT et l'APL. Ensuite (\$3.2.), les résultats ont été analysés, toutes cultures confondues (prairies exclues) pour s'affranchir de l'effet culture, sous un angle qualitatif ; l'objectif étant d'observer si le \%COT a un impact sur la qualification de l'APL (quatre classes, de bon à non conforme). Enfin, on étudie l'impact de la texture et du \%COT sur l'APL (exprimé en pourcent par rapport à la limite de conformité fixée pour la classe d'occupation de sol considérée). Pour cette dernière étude, seuls les sols cultivés sablo-limoneux (L), limoneux (A), limono-caillouteux (G) et argileux légers (E) ont été retenus, soit 1262 observations. Les sept autres classes texturales présentent des effectifs trop faibles et parfois des textures intermédiaires aux quatre classes précitées. Les résultats \%COT ont été répartis en quatre classes d'effectif équivalent dont les limites sont $0,998 \%$ ( $1^{\mathrm{er}}$ quartile), $1,150 \%$ (médiane) et $1,400 \%$ ( $3^{\mathrm{e}}$ quartile).

Les traitements statistiques (analyse de la variance, test de Tukey) des résultats sont effectués à l'aide du logiciel Minitab® 17.1.0.

\section{RÉSULTATS}

Le tableau 1 illustre la dispersion des résultats \%COT et APL, synthétisés par cultures (codées de C1 à C8). Logiquement, les \%COT les plus élevés sont observés en prairie (C8). Étant souvent situées au sud de la zone vulnérable (caractérisée par un \%COT plus élevé) et fréquemment intégrées dans des systèmes polyculturesélevage, les cultures de maïs et de colza présentent, par rapport aux autres cultures, des \%COT relativement élevés. À l'inverse, les parcelles de pommes de terre et légumes étant souvent situées dans des régions où les sols limoneux profonds prédominent, ces cultures sont régulièrement rencontrées dans des systèmes de polycultures avec moins de retour de matières organiques au sol.

En ce qui concerne l'APL, on observe que :

- les parcelles de betterave et la prairie présentent les valeurs les plus faibles ;

- la présence d'une CIPAN après une céréale réduit d'une vingtaine d'unités l'APL par rapport à une situation sans CIPAN (dans ce cas, la céréale est la plupart du temps suivie d'une autre céréale ou d'un colza) ;

- les valeurs plus élevées sont rencontrées dans les parcelles de pomme de terre.

\subsection{Relation \% COT - APL}

Les résultats ont été analysés en fonction de la culture récoltée (Tableau 2). Les analyses de variance (Dagnelie, 1999) indiquent des relations significatives $(\alpha=0,05)$ entre le \%COT et l'APL pour les céréales suivies d'une culture intermédiaire 
Tableau 1. Description des résultats d'analyse (pourcentage de carbone organique total [\%COT] et azote potentiellement lessivable [APL]) en fonction des classes de cultures - Nitrate nitrogen residue (APL) and percentage of total organic carbon (\%COT) results in function of crops classes.

\begin{tabular}{llrccccccc}
\hline Variable & $\begin{array}{l}\text { Classe de } \\
\text { culture }\end{array}$ & Effectif & Moyenne & $\begin{array}{l}\text { Écart- } \\
\text { type }\end{array}$ & Minimum & Quartile 1 & Mediane & Quartile 3 & Maximum \\
\hline \%COT & C1 & 94 & 1,2 & 0,3 & 0,7 & 1,0 & 1,1 & 1,3 & 2,3 \\
& C2 & 324 & 1,2 & 0,5 & 0,1 & 1,0 & 1,1 & 1,2 & 6,9 \\
& C3 & 335 & 1,3 & 0,8 & 0,5 & 1,0 & 1,2 & 1,4 & 13,2 \\
& C4 & 466 & 1,5 & 0,7 & 0,3 & 1,0 & 1,2 & 1,7 & 5,8 \\
& C5 & 136 & 1,1 & 0,3 & 0,7 & 0,9 & 1,1 & 1,2 & 4,2 \\
& C6 & 23 & 1,6 & 0,4 & 0,8 & 1,2 & 1,5 & 1,8 & 2,4 \\
& C7 & 9 & 1,2 & 0,2 & 0,7 & 1,0 & 1,2 & 1,3 & 1,4 \\
& C8 & 113 & 2,8 & 1,0 & 0,8 & 2,0 & 2,7 & 3,6 & 5,0 \\
\hline APL & C1 & 94 & 20,5 & 16,2 & 5,0 & 12,4 & 16,4 & 24,8 & 146,6 \\
& C2 & 324 & 46,0 & 31,1 & 3,0 & 21,8 & 39,6 & 62,4 & 193,0 \\
& C3 & 335 & 64,1 & 34,1 & 2,0 & 40,0 & 61,6 & 82,0 & 197,3 \\
& C4 & 466 & 62,7 & 47,0 & 3,9 & 32,3 & 49,8 & 77,0 & 523,2 \\
& C5 & 136 & 86,7 & 39,3 & 18,0 & 60,1 & 80,1 & 107,8 & 219,0 \\
& C6 & 23 & 70,1 & 27,9 & 15,9 & 49,2 & 70,0 & 90,0 & 126,0 \\
& C7 & 9 & 76,3 & 54,7 & 24,0 & 41,0 & 64,4 & 83,3 & 211,0 \\
& C8 & 113 & 10,4 & 9,0 & 1,0 & 4,0 & 8,0 & 13,8 & 44,5
\end{tabular}

$\mathrm{C} 1$ : betterave - sugar beet $; \mathrm{C} 2$ : céréale suivie d'une CIPAN - cereal followed by a catch crop ; $\mathrm{C} 3$ : céréale non suivie d'une CIPAN - cereal not followed by a catch crop ; 4 : maïs - corn ; 5 : pomme de terre - potato; 6 : colza d'hiver - winter oilseed rape $; \mathrm{C} 7$ : légume - vegetable $; \mathrm{C} 8$ : prairie - pasture.

Tableau 2. Relation entre le pourcentage de carbone organique total (\%COT) et l'azote potentiellement lessivable (APL) (régression et analyse de la variance) - Relation between nitrate nitrogen residue $(A P L)$ and percentage of total organic carbon (\%COT) (regression equations and analysis of variance).

\begin{tabular}{llll}
\hline $\begin{array}{l}\text { Classe de } \\
\text { culture }\end{array}$ & Équation de régression & $\mathbf{r}^{2}$ & $P$ \\
\hline C1 & APL $=7,24+11,49 \times \%$ COT & 0,04 & 0,063 \\
C2 & APL $=34,98+9,17 \times \%$ COT & 0,02 & 0,007 \\
C3 & APL $=65,79-1,13 \times \%$ COT & 0,00 & 0,628 \\
C4 & APL $=32,49+20,62 \times \%$ COT & 0,09 & 0,000 \\
C5 & APL $=72,6+12,79 \times \%$ COT & 0,01 & 0,193 \\
C6 & APL $=79,4-6,0 \times \%$ COT & 0,01 & 0,674 \\
C7 & APL $=128-44,4 \times \%$ COT & 0,03 & 0,651 \\
C8 & APL $=1,89+3,05 \times \%$ COT & 0,12 & 0,000 \\
\hline
\end{tabular}

piège à nitrate (CIPAN), le maïs et les prairies. Dans ces trois situations, le coefficient de détermination reste cependant relativement faible $(\approx 0,1)$. Ceci n'est cependant pas illogique puisque l'APL est avant tout un indicateur de la gestion de l'azote mise en œuvre par l'agriculteur, comme Fonder et al. (2010) et Heens (2013) l'ont montré.

Pour la betterave, la pomme de terre, le colza et les céréales non suivies d'une CIPAN, la relation entre le $\%$ COT et l'APL n'est pas significative.

\subsection{Relation \%COT - qualification de l'APL}

L'analyse de la variance des 1362 observations indique l'existence d'une différence significative $(p=0,000)$ de $\%$ COT en fonction de la qualification du résultat; les parcelles qualifiées «non conformes » présentant un \%COT plus élevé que les autres parcelles (Figure 3).

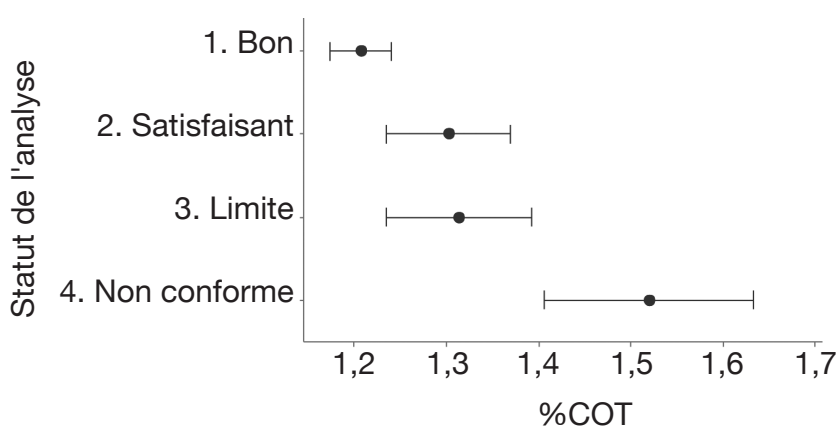

Figure 3. Intervalle de confiance du pourcentage de carbone organique total (\%COT) moyen par qualification du résultat de l'azote potentiellement lessivable (APL) - Interval plot of total organic carbon (\%COT) in relation with nitrate nitrogen residue (APL) qualification. 


\subsection{Relation \%COT - propriétés édaphiques - $\%$ APL}

L'analyse de la variance (GLM avec comme facteurs, la texture et la classe de \%COT) a mis en évidence des différences significatives $(P=0,000)$ entre les valeurs APL (exprimées en pourcent par rapport à la limite de non-conformité fixée pour la classe d'occupation de sol considérée) dans les différentes classes de COT et pour les différentes textures du sol. Un test de Tukey permet de préciser ces différences : les textures L et A présentent des APL plus proches des limites de nonconformité que les textures $\mathrm{E}$ et $\mathrm{G}$ (respectivement $84 \%, 77 \%, 61 \%$ et $53 \%$ pour les classes L, A, E et $\mathrm{G})$. En ce qui concerne le \%COT, les classes 2 et 3 (valeurs \%COT comprises entre les quartiles 1 et 3 ) sont significativement différentes des classes 1 et 4 (respectivement 52\%,67\%,69\% et $87 \%$ pour les classes 1,2, 3 et 4$)$.

La même analyse de variance révèle également l'absence d'interaction entre le \%COT et la texture, telle que l'illustre la figure 4. L'importance de l'intervalle de confiance pour les associations E-2 et G-3 s'explique par des résultats très disparates et peu nombreux (respectivement trois et six observations).

La prise en compte des propriétés hydromorphiques des sols n'a pas permis de mieux expliquer les résultats APL et les interactions entre facteurs explicatifs.

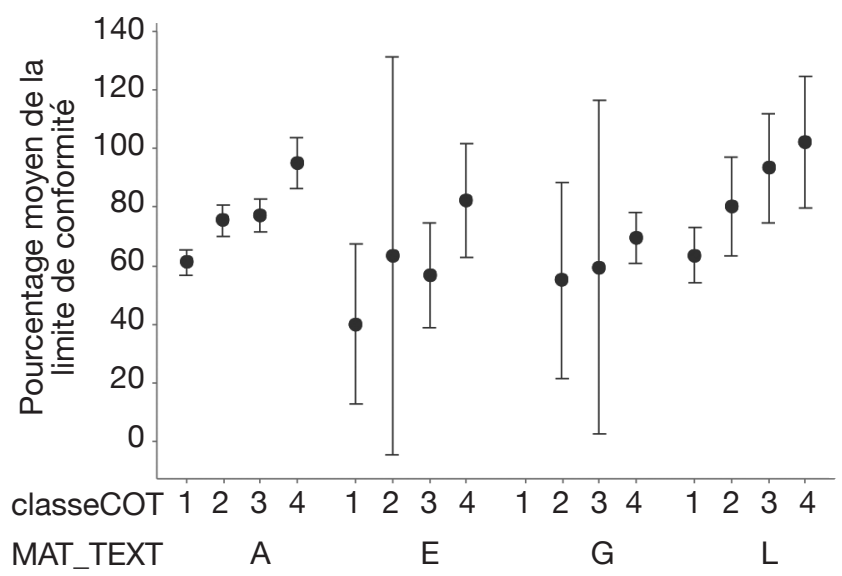

Figure 4. Intervalle de confiance du pourcentage moyen de l'azote potentiellement lessivable (APL) par rapport à la limite de non-conformité, par pourcentage de carbone organique total (\%COT) et par texture - Confidence interval of the nitrate nitrogen residue (APL) (in \% of the noncompliance limit) by total organic carbon (\%COT) and by soil texture.

classeCOT $1=<0,998 \%-<0.998 \% ; 2=<1,150 \%-$ $<1.150 \%, 3=<1,400 \%-<1.400 \% ; 4=>1,400 \%-$ $>1.400 \%$; MAT_TEXT A : limoneux - silt ; L : sablolimoneux - sandy loam; $\mathrm{E}$ : argile légère - clay loam ; G : limono-caillouteux - stony loam.

\section{DISCUSSION}

L'examen des résultats d'analyses pratiquées sur 1362 échantillons de sol a mis en évidence l'existence d'une relation significative entre l'APL et le \%COT pour trois cultures : plus le \%COT est élevé, plus l'APL est élevé. Des observations similaires ont été mises en évidence par Geypens et al. (2005) : seulement $38 \%$ des parcelles à taux de carbone élevé présentaient un APL inférieur au seuil de $90 \mathrm{~kg} \mathrm{~N}-\mathrm{NO}^{3} \cdot \mathrm{ha}^{-1}$, alors que $78 \%$ des parcelles à taux de carbone faible présentaient un APL inférieur à ce seuil. Notons d'une part que pour les cinq autres cultures, la relation n'est pas significative et que pour toutes les cultures, les coefficients de détermination sont très faibles, indiquant que d'autres facteurs doivent expliquer plus largement le résultat APL. Parmi ces facteurs, la fertilisation (minérale et/ou organique) constitue l'élément explicatif principal du résultat APL (Beaudoin et al., 2005). D'autres facteurs peuvent également expliquer l'APL, tels que la qualité du semis (date et méthode de semis) d'une CIPAN après récolte de céréale (De Toffoli et al., 2010 ; Justes et al., 2012 ; Constantin et al., 2015) ou la gestion d'une parcelle entre la récolte du colza et le semis du froment (travail régulier du sol pour gestion des adventices et ravageurs versus non-travail du sol pour favoriser les repousses de colza) (Reau et al., 2006) .

Par ailleurs, chaque résultat APL est qualifié (de bon à non conforme) après comparaison à des références établies annuellement (pour tenir compte de l'année climatique) par culture. Pour une exploitation contrôlée, plus d'un résultat sur trois non conformes implique l'entrée dans un programme d'observation, voire des sanctions financières. L'analyse statistique réalisée a mis en évidence une relation significative entre la qualification d'un résultat APL et le \%COT : les parcelles non conformes présentent un \%COT supérieur aux autres parcelles.

La prise en compte de propriétés édaphiques met en évidence que l'APL moyen est plus proche de la limite de non-conformité pour les textures limoneuses et argileuses que pour les textures sablo-limoneuses ou limono-caillouteuses. À texture identique, l'APL moyen est également plus proche de la limite de non-conformité lorsque la teneur en COT augmente. L'analyse de la variance n'a cependant pas révélé d'interaction entre ces deux facteurs (\%COT et texture).

\section{CONCLUSIONS}

Cette étude met donc en évidence que le sol et principalement sa teneur en carbone organique total constituent un facteur de risque d'APL élevé et, par conséquent, de contamination des eaux souterraines par le nitrate. Rappelons que cette étude s'appuie sur 
les résultats d'un contrôle APL réalisé dans toute la zone vulnérable de la région wallonne et que, dans le cadre de ce contrôle, les fertilisations (minérales et/ ou organiques) appliquées par les agriculteurs ne sont pas connues. Or, comme mentionné précédemment, la gestion des apports azotés constitue l'un des principaux facteurs d'APL élevé.

$\mathrm{Au}$ vu de ces résultats, il est recommandé, d'une part, de s'assurer que la teneur en carbone organique total soit correctement prise en compte dans les conseils de fertilisation azotée qui peuvent être prodigués aux agriculteurs et, d'autre part, de sensibiliser ceux-ci à bien intégrer ce paramètre dans la gestion de leurs engrais azotés (minéraux et organiques) et de leurs cultures pièges à nitrate (assurer une mise en place correcte du couvert pour bien capter l'azote nitrique produit lors de la minéralisation automnale).

\section{Remerciements}

Les auteurs adressent leurs remerciements au Service public de Wallonie (DGO3) pour le financement de l'étude.

\section{Bibliographie}

Bah B.B. et al., 2007. Légende de la Carte Numérique des sols de Wallonie. Version 2. Gembloux, Belgique: Université de Liège - Gembloux Agro-Bio Tech.

Beaudoin N. et al., 2005. Nitrate leaching in intensive agriculture in Northern France: effect of farming practices, soils and crop rotations. Agric. Ecosyst. Environ., 111, 292-310.

Brahy V.et al.,2014. Les indicateurs clés de l'environnement wallon 2014. Namur, Belgique: Service public de Wallonie.

Bremner J.M., 1965. Inorganic forms of nitrogen. In: Black C.A., ed. Methods of soils analysis. Part 2. Madison, WI, USA: American Society of Agronomy, 1179-1237.

Bronick C.J. \& Lal R., 2005. Soil structure and management: a review. Geoderma, 124(1-2), 3-22.

Buysse P.etal.,2013. Fifty years of crop residue management have a limited impact on soil heterotrophic respiration. Agric. For. Meteorol., 180, 102-111.

Chenu C., Le Bissonnais Y. \& Arrouays D., 2000. Organic matter influence on clay wettability and soil aggregate stability. Soil Sci. Soc. Am. J., 64(4), 1479-1486.

Colinet G. et al., 2005. Base de données sols de Requasud. $2^{\grave{e}}$ synthèse. Gembloux, Belgique : Asbl Requasud.

Constantin J., Le Bas C. \& Justes E., 2015. Large-scale assessment of optimal emergence and destruction dates for cover crops to reduce nitrate leaching in temperate conditions using the STICS soil-crop model. Eur. J. Agron., 69, 75-87.
Dagnelie P., 1999. Statistique théorique et appliquée. Tome 2. Inférence statistique à une et deux dimensions. $2^{e}$ édition. Bruxelles : De Boeck Université.

D'Hose T. et al., 2014. The positive relationship between soil quality and crop production: a case study on effect of farm compost application. Appl. Soil Ecol., 75, 189-198.

De Toffoli M., Bontemps P.-Y. \& Lambert R., 2010. Synthèse de résultats d'essais de cultures intermédiaires pièges à nitrate à l'Université catholique de Louvain. Biotechnol. Agron. Soc. Environ., 14(S1), 79-89.

Deneufbourg M., Vandenberghe C., Heens B. \& Marcoen J.M., 2013a. Suivi de la lixiviation du nitrate en plein champ par la technique lysimétrique : retour de huit années d'expérience. Biotechnol. Agron. Soc. Environ., 17(S1), 177-186.

Deneufbourg M. et al., 2013b. Mise en œuvre du Programme de Gestion Durable de l'Azote à l'échelle de deux petits bassins versants (Arquennes - Belgique) et évaluation d'impact par le suivi des flux de nitrate aux exutoires et par modélisation numérique. Biotechnol. Agron. Soc. Environ., 17(S1), 164-176.

Destain J.-P., Fonder N., Xanthoulis D. \& Reuteur V., 2010. L'isotope stable $15 \mathrm{~N}$ et le lysimètre, des outils complémentaires pour l'étude de la lixiviation de l'azote dans les sols agricoles. Biotechnol.Agron. Soc. Environ., 14(S1), 91-96.

Fenoll J. et al., 2011. Reduction of the movement and persistence of pesticides in soil through common agronomic practices. Chemosphere, 85(8), 1375-1382.

Fenoll J. et al., 2014. Assessment of agro-industrial and compost organic wastes for reducing the potential leaching of triazine herbicide residue through the soil. Sci. Total Environ., 493, 124-132.

Fonder N., Heens B. \& Xanthoulis D., 2010. Optimisation de la fertilisation azotée en cultures industrielles légumières sous irrigation. Biotechnol. Agron. Soc. Environ., 14(S1), 103-112.

Genot V. et al., 2012. Base de données sols de Requasud. $3^{\grave{e}}$ synthèse. Gembloux, Belgique : Asbl Requasud.

Geypens M., Mertens J., Ver Elst P. \& Bries J., 2005. Evaluation of fall residual nitrogen influenced by soil chemical characteristics and crop history in Flanders (Belgium). Commun. Soil Sci. Plant Anal., 36(1-3), 363372.

Grignani C., Zavattaro L., Sacco D. \& Monaco S., 2007. Production, nitrogen and carbon balance of maize-based forage systems. Eur. J. Agron., 26(4), 442-453.

Guiot J., 1975. Estimation des réserves azotées du sol par détermination de l'azote minéral. Rev. Agric., 28, 11171132.

Heens B., 2013. Fertilisation azotée des légumes industriels. Biotechnol. Agron. Soc. Environ., 17(S1), 207-214.

ISO 10694, 1995. NBN ISO 10694: qualité du soldosage $d u$ carbone organique et du carbone total après combustion sèche (analyse élémentaire). Genève, Suisse : ISO. 
ISO 14235, 1998. NBN ISO 14235 : qualité du sol - dosage du carbone organique par oxydation sulfochromique. Genève, Suisse : ISO.

Justes E. et al., 2012. Réduire les fuites de nitrate au moyen de cultures intermédiaires: conséquences sur les bilans d'eau et d'azote, autres services écosystémiques. Rapport d'étude. Versailles-Grignon, France : INRA.

Maltas A., Charles R., Jeangros B. \& Sinaj S., 2013. Effect of organic fertilizers and reduced-tillage on soil properties, crop nitrogen response and crop yield: results of a 12-year experiment in Changins, Switzerland. Soil Tillage Res., 126, 11-18.

Mukherjee I., 2009. Effect of organic amendments on degradation of atrazine. Bull. Environ. Contam. Toxicol., 83(6), 832-835.

Nevens F. \& Reheul D., 2005. Agronomical and environmental evaluation of a long term experiment with cattle slurry and supplemental inorganic $\mathrm{N}$ applications in silage maize. Eur. J. Agron., 22(3), 349-361

Reau R., Bouthier A. \& Champolivier L., 2006. La lixiviation d'azote nitrique dans les rotations céréalières avec colza : un diagnostic à partir de l'analyse de résultats d'expérimentations pluriannuelles et de modélisations. Ol. Corps Gras, Lipides, 13(6), 403-412.

Vanden Nest T. et al., 2014. Effect of organic and mineral fertilizers on soil $\mathrm{P}$ and $\mathrm{C}$ levels, crop yield and $\mathrm{P}$ leaching in a long term trial on a silt loam soil. Agric. Ecosyst. Environ., 197, 309-317.

Vandenberghe C., Mohimont A.C., Garot T. \& Marcoen J.M., 2004. The agricultural area survey as a tool for implementing the European Nitrates Directive in the Walloon Region of Belgium. In: Hatch D.J., Chadwick D.R., Jarvis S.C. \& Roker J.A. Controlling nitrogen flows and losses. Wageningen, The Netherlands: Wageningen Academic Publishers, 93-96.

Vandenberghe $C$. et al., 2005. Implementation of the Nitrate Directive in Belgium: the agricultural surface survey. In: Schröder J.J. \& Neeteson N. Management in agrosystems in relation to the Water Framework Directive. Wageningen, The Netherlands: Plant Research International, 332-334.

Vandenberghe C., 2010. Mise en relation de l'évolution de l'agriculture et de la qualité de l'eau entre 1950 et 2000. Biotechnol. Agron. Soc. Environ., 14(S1), 9-16.

Vandenberghe C., Colinet G., Destain J.-P. \& Marcoen J.M., 2013a. Mise en œuvre de la révision du Programme d'action lié à la Directive Nitrates. Biotechnol. Agron. Soc. Environ., 17(S1), 161-163.

Vandenberghe C. et al., 2013b. Contrôle de l'azote potentiellement lessivable dans le sol en début de période de lixiviation. Établissement des valeurs de référence. Biotechnol. Agron. Soc. Environ., 17(S1), 231-236.

Vandenberghe C. \& Colinet G., 2014. Estimation of the soil nitric nitrogen concentration at plot scale. Impact of the sampling density on the result's precision. Poster session presented at Day of the Young Scientists, 24 February 2014, Bruxelles, Belgique, http://hdl.handle. net/2268/164474, (26.10.2016).

Whitmore A.P., Bradburg N.J. \& Johnson P.A., 1992. Potential contribution of ploughed grassland to nitrate leaching. Agric. Ecosyst. Environ., 39(3-4), 221-233. 